\title{
Behavior of RC-slabs under impact-loading
}

\author{
Tino Kühnª and Manfred Curbach \\ TU Dresden, Institute of concrete structures, 01069 Dresden, Germany
}

\begin{abstract}
In this paper the current state of a research project on the behavior of reinforced concrete slabs subjected to impact loads are presented. As one part of the project several slabs were tested under varying conditions. As a result there failure behavior is analyzed and parameter dependences are studied. Variations like the drop height and drop mass and there substituted drop velocity and energy, the influence of the concrete strength and the level of reinforcement are part of the full project. Additional parameters like impactor size and shapes are evaluated in peripheral. Due to the large number of slabs and parameters combination a consistent database will be created for later benchmark tests and calibration of prediction models. The presented paper gives some insight in the methodology, the problems which need to be solved and first principal example results for public discussion.
\end{abstract}

\section{Principles}

The behavior of concrete slabs subjected to dynamic loading are experimentally and numerically investigated at the Institute of Concrete Structures, TU Dresden. The projects are focused on the local material behavior at different loading conditions as well as on their influence on the global structural behavior caused by impact loads such as rock fall or vehicle impact at moderate strain velocities. The higher velocity in dynamic experiments leads to assumed strain rate effects of the concrete material which is verified by many authors with different significance within the compressive [1] and tensile [2] domain. Nevertheless of the structural influences this effect can be described by numerical models for example as a viscous effect at low rates or a crack retardation effect at higher velocities [3]. With numerical benchmarks of standard experiments it could be shown that the structural and material response could not really separated and therefore the results are difficult to transfer to other structures [4]. As shown by experimental drop tests in [5] the knowledge of the detailed behavior of the sample interaction with the test facility is crucial to achieve best and publishable results. The influence of the material is somehow recognizable but not really to separate. To understand the dynamic behavior numerical simulation even with rough material assumptions are indispensable which gives more local insight and a feeling for the global reaction without disturbance [6]. In this work sample experiments on reinforced concrete slabs using a newly build high energy drop tower are presented as a subset of the underlying projects aims. This paper gives short introductions to the experimental facility with reflecting basic experimental results.

${ }^{a}$ Corresponding author: tino.kuehn@tu-dresden.de

\subsection{The experimental facility}

Concerning those requirements, a high energy drop tower was newly designed and implemented at the Otto Mohr Laboratory in Dresden. With a drop height up to 11 meters and a designed drop mass of up to 2.5 tons, a free fall energy of approximately $200 \mathrm{~kJ}$ can be used testing sample structures with a geometry of approx. $2 \times 2$ meters. The moderate impact velocity of up to $15 \mathrm{~m} / \mathrm{s}$ in combination with a variable impactor system spans a wide dynamic load range useful for experiments with pure compressive to pure spall failure modes.

In addition a pneumatic accelerometer pipe can be vertically included in the 4 stories building. Two parallel pneumatic reservoirs with a capacity of 10001 can be loaded up to 16 bar of pressure which represents the nearly same energy storage of $200 \mathrm{~kJ}$. The pipe diameter is fixed to $100 \mathrm{~mm}$. A variety of projectiles up to designed $100 \mathrm{~kg}$ can be included and accelerated up to the velocity of $150 \mathrm{~m} / \mathrm{s}$. With this facility high dynamic impact problems can be investigated. Material modelling and model validation as well as specific optimization or design changes for protective wall layers or other such structures might be performed. In order to have a wide range of experimental setup a $3 \times 3 \mathrm{~m}$ flexible span was designed. The underlying shock absorbing mass of 30 tons is mounted on four air springs with a maximum amplitude of $500 \mathrm{~mm}$ and adjustable height and stiffness.

Figure 1 gives an impression of the setup area with a destroyed $1.5 \times 1.5 \mathrm{~m}$ slab sample, the drop carriage with a $100 \mathrm{~mm}$ diameter impactor and the vertical guiding girders to the left and right side. Beside the impactor in the middle, the booth rescue shock dampers can be seen which are activated after punching through the specimen to adsorb excessive energy and prevent further destruction. 


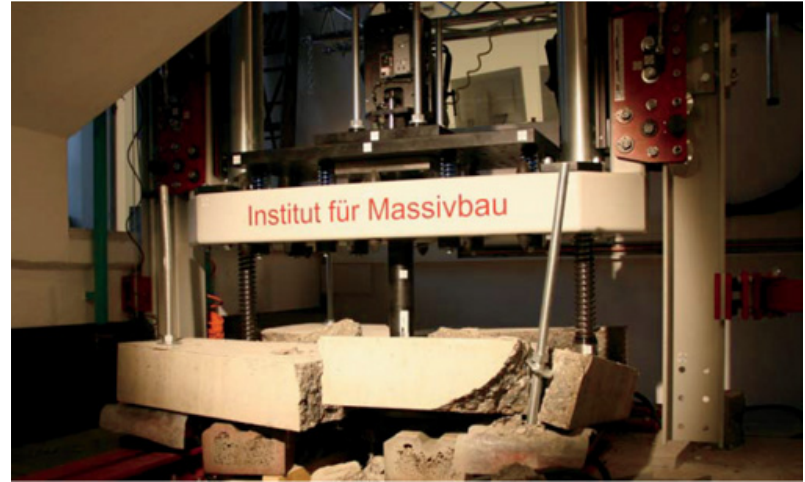

Figure 1. view to the setup field with drop carriage and damaged sample.

\subsection{Energy and impulse relations}

Static experiments are force or displacement controlled in most cases. Free fall drop tower tests are energy driven and can be controlled by the drop height and the falling mass. Energy $E$ is simply related to the potential energy at specified high $h$ as $E=m \cdot g \cdot h$ with Mass $m$ and $g$ as natural acceleration. Ignoring frictional effects the impact velocity $v$ is given by:

$$
v=\sqrt{2 \cdot g \cdot h}
$$

whereas the free fall time $t$ is given by:

$$
t=\sqrt{\frac{2 \cdot h}{g}}
$$

The impulse induced by the impactor while hitting the slab is partly transferred to the specimen. Ignoring surface effects leads to a resultant impactor velocity $v_{I R}$ of:

$$
v_{I R}=k \cdot \frac{m_{I} \cdot v_{I}+m_{S} \cdot v_{S}-m_{S} \cdot\left(v_{I}-v_{S}\right)}{m_{I}+m_{S}} .
$$

The resultant specimen velocity $v_{S R}$ is given by:

$$
v_{S R}=k \cdot \frac{m_{I} \cdot v_{I}+m_{S} \cdot v_{S}-m_{I} \cdot\left(v_{S}-v_{I}\right)}{m_{I}+m_{S}}
$$

with $\mathrm{k}$ indicating the impulse transfer between 1 (elastic) and 0 (plastic), the masses $m$ and the initial velocity $v$ of the specimen and impactor in each case. The experimentally determined coefficient of restitution $k=$ 0.1 for example, qualifies an experimental standard setup of $2 \mathrm{~m}$ drop height with a $590 \mathrm{~kg}$ drop mass hitting a C40 concrete slab of $1.5 \times 1.5 \times 0.3$ meters. This leads to a small backflip of the impactor with the velocity $v_{I R}=-0.48 \mathrm{~m} / \mathrm{s}$ and a resultant foundation velocity of $v_{I S}=0.14 \mathrm{~m} / \mathrm{s}$. Thus the measurement of the foundations seismic mass velocity gives a first integral value for a global damage behavior description of the specimen.

\subsection{Foundation and springs}

The foundation is mounted on 4 double gaiter air springs with variable pressures and several stabilizers. In this

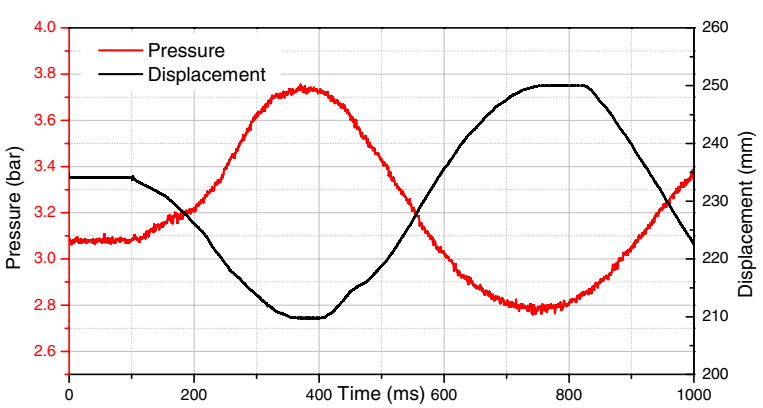

Figure 2. Pressure and displacement distribution of the accelerated foundation.

example the mass displaces $24 \mathrm{~mm}$ within a time of approx. $200 \mathrm{~ms}$. The spring behavior is highly nonlinear and depends on the geometrical change at different strokes and is pressure dependent as well. This leads to a static pressure load of $p_{S}=3.1 \mathrm{bar}$ at $235 \mathrm{~mm}$ stroke length to carry the foundation, payload and specimens mass with a force of $80 \mathrm{kN}$ for each spring. Due to the dynamic load the additional amplitude of $5.5 \mathrm{kN}$ is calculated from the mass acceleration for each spring. The air system is closed during the testing so that force changes lead to air compression within the system. In this case the isentropic pressure-volume relation is given by:

$$
p_{D}=\frac{p_{S}\left(h_{S}\right) \cdot V_{S}\left(h_{S}\right)^{\kappa}}{V_{D}^{\kappa}}
$$

with the pressure $p_{S}\left(h_{S}\right)$, the spring volume $V_{S}\left(h_{S}\right)$ at static conditions and $p_{D}$ and $V_{D}$ the dynamic response. The parameter $\kappa$ is the isentropic exponent with a value of $\kappa=1.4$ for dry air. In the given example the pressure increases to $p_{D}=3.7$ bar with a stroke length of $210 \mathrm{~mm}$. The temperature within the spring increases during compression. Assuming adiabatic condition the relation is given as follows:

$$
T_{D}=\frac{T_{S}}{\left(\frac{V_{D}}{V_{S}}\right)^{\kappa}}-T_{S}
$$

with $T_{S}$ and $T_{D}$ the temperature at static and dynamic load conditions. For the sample experiment the temperature increase is given by $41 \mathrm{~K}$ assuming 20 degree ambient temperature. The volumetric work during compression is given by:

$$
d W=\frac{-p_{S} \cdot V_{S}}{\kappa-1} \cdot\left(1-\left(\frac{V_{S}}{V_{D}}\right)^{\kappa-1}\right)
$$

For the sample experiment it is given as $1.8 \mathrm{~kJ}$ for each spring which means, that approx. $65 \%$ of the drop energy is transferred to the springs. Figure 2 illustrates the measured foundation data from the sample experiment which fit well to the analytical assumption. The knowledge about the reaction, and the adjustable foundation stiffness could be a first indicator for a global experimental judgment.

\subsection{Second hit prevention}

Since the foundation follows Eq. (4), the impactor reaction velocity can be calculated with Eq. (3) where an increasing 


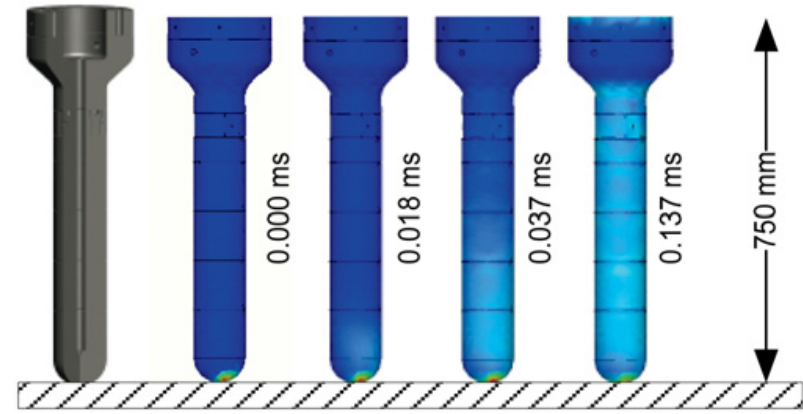

Figure 3. Segmented Impactor, stress state at different timesteps.

coefficient of restitution leads to a backflip velocity increase in negative direction. In the sample experiment the negative velocity $v_{I R}$ leads to impactor reversal followed by a second (or more) hit onto the sample. To prevent this from the study the impactor carrier can be automatically stopped with self-locking breaks exactly at the upper death point of the movement ad zero forces.

\subsection{Impactors}

Following this approach just the first hit should be relevant for the stiffest experiment. The length of this hit is given by the length of the impactor assuming a linear elastic compression and expansion. The time is given by:

$$
t_{I}=2 \frac{l_{I}}{c_{I}}
$$

with the impactor length $l_{I}$ and the impactor sound speed $c_{I}$ given by:

$$
c_{I}=\sqrt{\frac{E \cdot(1-\mu)}{\rho \cdot\left(1-\mu-2 \cdot \mu^{2}\right)}},
$$

the elastic modulus $E$ of steel, its poison ratio $\mu$ and its density $\rho$. With a sound speed of approx. $c_{I}=$ $5500 \mathrm{~mm} / \mathrm{ms}$, the impulse duration for this example is given with $t_{I}=0.274 \mathrm{~ms}$. Further assuming purely elastic undamaged response, the maximum stress can be calculated independently of the mass by:

$$
S_{I}=\frac{1}{2} \cdot \rho \cdot c_{I} \cdot v_{I}
$$

The impactor stress for this sample experiment is $S_{I}=$ $134 \mathrm{MPa}$ which is simply correlated to the impactor force of $F_{I}=1.1 \mathrm{MN}$ on its relevant area.

Figure 4 compares the measured impactor forces during the first $4 \mathrm{~ms}$ given by piezoelectric strain sensors at the top and semiconductor based strain gages at the bottom. The maximum force of approx. $0.8 \mathrm{MN}$ is less than the theoretical assumed value. The impactor frequency can be found at the top curve as overlaid oscillation of the nearly $1 \mathrm{~ms}$ mean impulse. The stiffness of the impactor system can be modified to extent the load duration and impulse shape. This is done by additional layers between impactor and drop masses and adjustable springs.

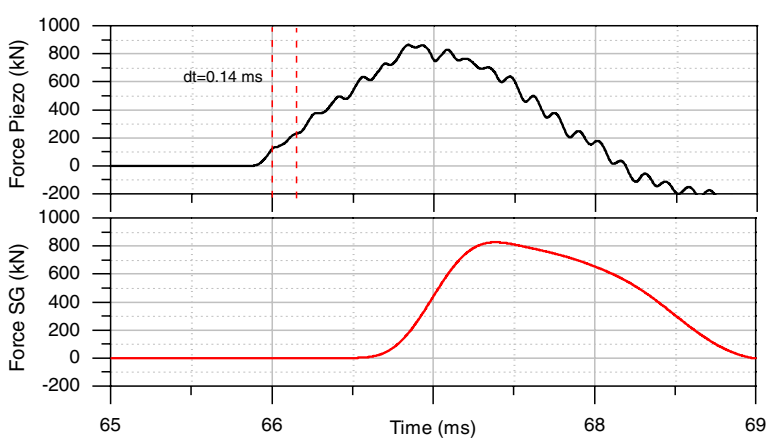

Figure 4. Sample impactor forces for piezoelectric and resistance based sensor types.

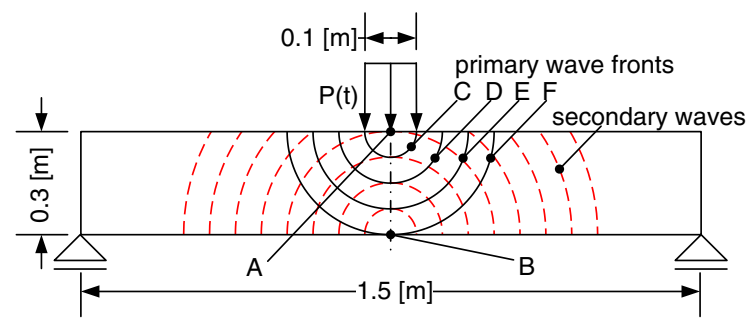

Figure 5. Principal sketch of the wave propagation in the slab during the first few milliseconds.

\section{Principle slab behavior}

Until this point the outer system was considered with abstracting the sample slab behavior into the coefficient of restitution $k=0.1$ as the root point of investigations. The slab is mounted on the foundation mass with four solid bearings which are also functioning as load cells. This gives a stiff linkage between sample and basement. Comparing Fig. 2 and Fig. 4, the load impulse finished at approx. $70 \mathrm{~ms}$ while the foundation movement starts $100 \mathrm{~ms}$ later. Hence the bearings can assumed to be fixed in vertical direction during the interesting inspection time.

Figure 5 sketches the load conditions during the first load impulse from the upper center point A. With equation (9) the longitudinal wave speed for the concrete sample is given by $\mathrm{c}_{\mathrm{CL}}=4025 \mathrm{~mm} / \mathrm{ms}$. With a slab thickness of $H_{C}=300 \mathrm{~mm}$, the time for the first wave reflection is given by $t_{\mathrm{CL}}=0.075 \mathrm{~ms}$. At this time the pressure wave reaches the lower point $B$ and reflects back as a tensile wave.

The numerically computed vertical and horizontal stress components in the top position (A) and bottom position (B) are given as a function of time at Fig. 6. As the wave front spreads spherically within the slab the stress values decrease with increasing distance $r$ from the upper side roughly according to $\mathrm{r}^{-1}$. After the initial phase a reflection of stresses arises at the bottom side with a reversal of compression into tension. The reflected wave front interferes with the incoming wave and the stress state within the structure becomes more complex. Thus, the local strain rate values will span from a high level at the impacted zone to a low level distributed over the whole structure.

The vertical displacements of position (A) and (B) are shown in Fig. 7. Damage or plasticity was not allowed in 


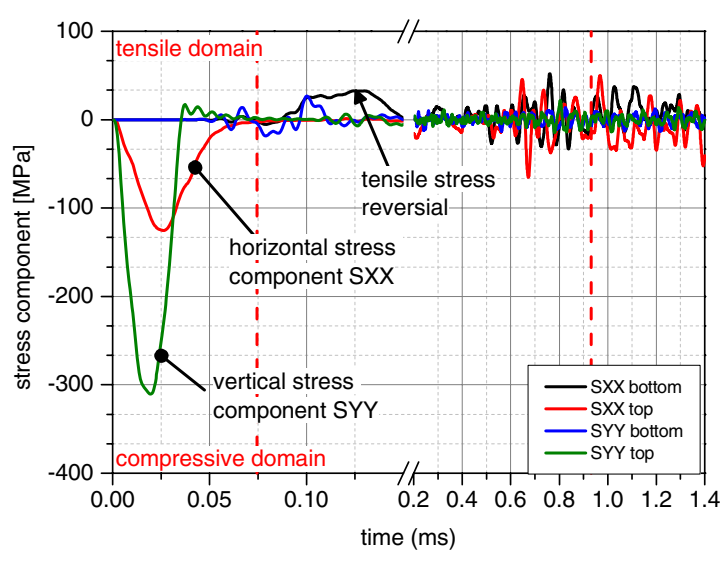

Figure 6. Computed stress distribution during the first $1.4 \mathrm{~ms}$.

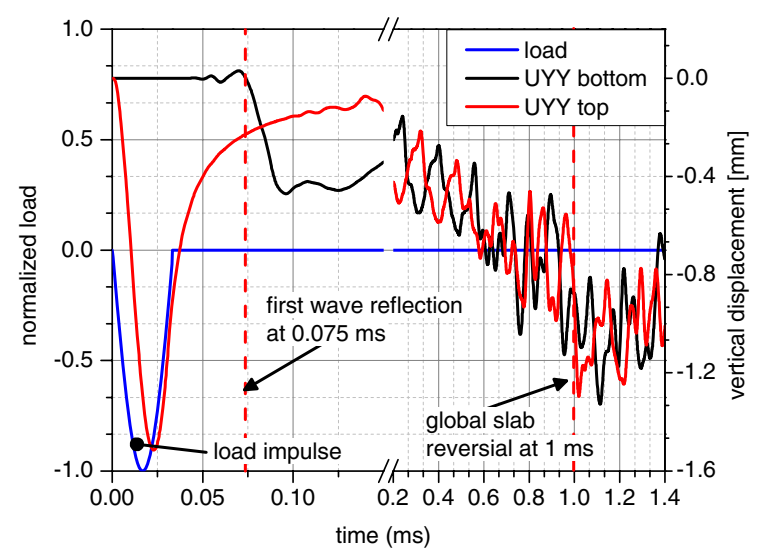

Figure 7. Computed displacement distribution during the first $1.4 \mathrm{~ms}$.

this principal simulation. An indentation effect can be seen during the loading time with much larger displacement of (A) compared to (B). The indentation relieves after the end of the loading time until (A) and (B) have the same displacement values superposed by oscillations. A global deflection goes on with a maximum value at approximately $\mathrm{t}=1 \mathrm{~ms}$, which corresponds to the natural period of this eigenmode.

Resuming Fig. 4 the impulse load duration fits well with the global slab displacement time which could lead to more static like behavior and tensile bending cracking.

\section{Experimental results}

The investigation aims to deliver robust experimental reference datasets of impacting slabs under different conditions according to the following questions:

- How is the influence of the impact velocity,

- the influence of the mass and its related energy,

- the scale influence of the sample and impactor geometry,

- the influence of the rebar placement and design

- and the strength influence of different concrete grades?

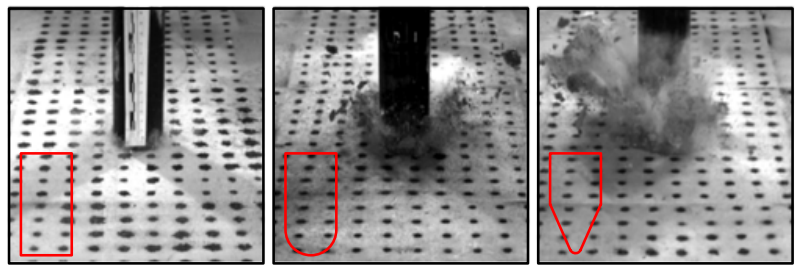

Figure 8. Impactor shape influence on the crushing behavior at the same time step.

\subsection{Experimental setup}

Beginning with a reference state several parameters are varied with respect to a statistically significant accuracy. Reference data is given:

- Sample

- Concrete grade: $\mathrm{C} 40$

$\circ$ Geometry: $1.5 \times 1.5 \times 0.3 \mathrm{~m}$

- Reinforcement Type BST500, D $10 \mathrm{~mm}$

- $2 \times 100 \times 100 \mathrm{~mm}$ with $25 \mathrm{~mm}$ wall distance

- Impactor
$\circ$ Drop height $2 \mathrm{~m}$
- Drop weight $800 \mathrm{~kg}$
- Impactor length $750 \mathrm{~mm}$
- Impactor diameter $100 \mathrm{~mm}$
- Flat impactor shape

- Foundation

○ Weight plus payload $32.000 \mathrm{~kg}$

- Position $235 \mathrm{~mm}$ at 3.1 bar pressure

Standard data records are available for the impactor forces and its tip acceleration where forces are simultaneously measured with different sensor types. Foundation behavior is recordable with its displacement, acceleration measure and spring pressure distribution. The sample reaction forces to the foundation are measured at four bearing points with three types of sensors to observe low static changes as well as fast dynamic changes at the same position.

The data is usually recorded with a sampling rate of $2 \mathrm{MHz}$ for the relevant time of approx. 1 second. In addition two high speed cameras are triggered to directly measure displacements as needed. In the following a subset of principal experimental results is shown without the claim to completeness. The underlying project and data acquisition process is still running and experimental improvements and data analysis are continuous tasks.

\subsection{The Impactor shape influence}

The shape of the impactors tip plays a major role for the damage behavior of the slab sample. In the following it was varied at reference conditions with impactor diameter $100 \mathrm{~mm}$ and slab thickness $200 \mathrm{~mm}$. Figure 8 compares the crushing behavior by snapshots at $100 \mathrm{~ms}$ post triggering. The impactors already intruded approx. $100 \mathrm{~mm}$ in each case and one can distinguish between two principal failure modes. The flat tip leads to a punching like failure while the round and sharp shape tip causes crushing at the top surface. 


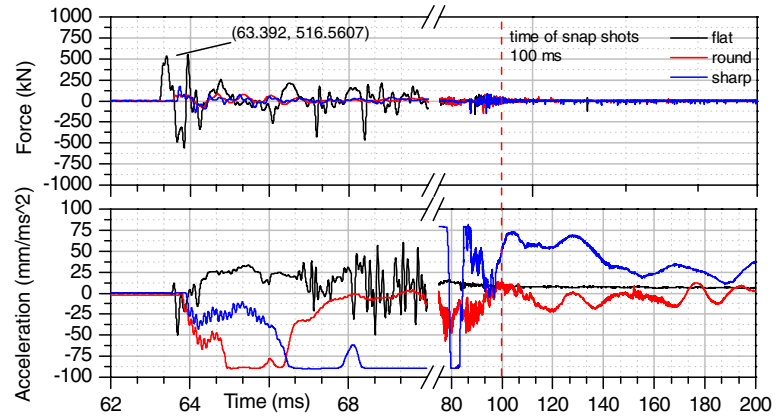

Figure 9. Impactor force and acceleration distribution for different tip shapes.

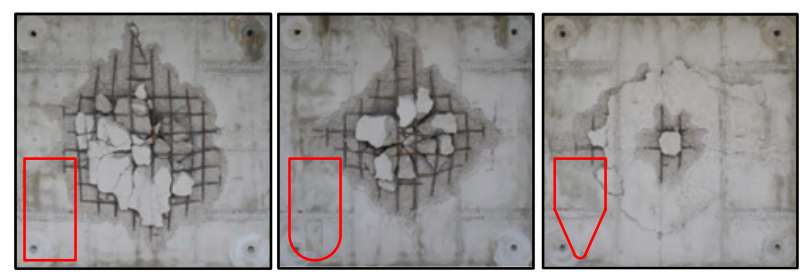

Figure 10. Impactor shape influence to the damage behavior on the slabs rear side.

Taking a closer look to the impactor forces at Fig. 9 shows a high value of its compressive maximum at $534 \mathrm{kN}$ for the flat, $187 \mathrm{kN}$ for the round and $152 \mathrm{kN}$ for the sharp shaped tip. The acceleration distributions within the tip are low for the flat tip with $4000 \mathrm{~g}$ and exceed the sensors ultimate value of $5.000 \mathrm{~g}$ in the other cases. More interesting than the maximum values is their further distribution. While the flat tip values are nearly zeroed after approx. $10 \mathrm{~ms}$, the round and sharp values are still higher, indicating the still ongoing crushing process.

The process finishes with the impactor fully punched through the slab for the flat, and sticking for the other cases. Figure 10 compares the slabs rear sides where the punched cone can be recognized to the left and totally different shapes are found at the right side.

\subsection{Drop mass variation}

The drop mass was extended to $1800 \mathrm{~kg}$ with respect to the reference configuration with flat impactor tip. The slab thickness was again reduced to $200 \mathrm{~mm}$. The drop energy increases from $15 \mathrm{~kJ}$ to $35 \mathrm{~kJ}$. The resultant impactor forces and the acceleration distributions are shown in Fig. 11.

The force maximum of the first relevant impulse increases from $534 \mathrm{kN}$ to $671 \mathrm{kN}$ which is moderate according to the energy increase but without any statistical consideration. This is due to the fact that the slab sample is already totally damaged by the lighter drop mass. Totally damaged means at this point, that the impactor punches through the whole thickness of the slab and only the underlying rebar layer is carrying the load. With higher energy this additional amount of energy leads to deeper penetration until the rescue dampers adsorb this to prevent further demolition. The energy absorption by the foundation increases from approx. $11 \mathrm{~kJ}$ to $17 \mathrm{~kJ}$ but the ratio of the introduced drop energy reduces from approx.

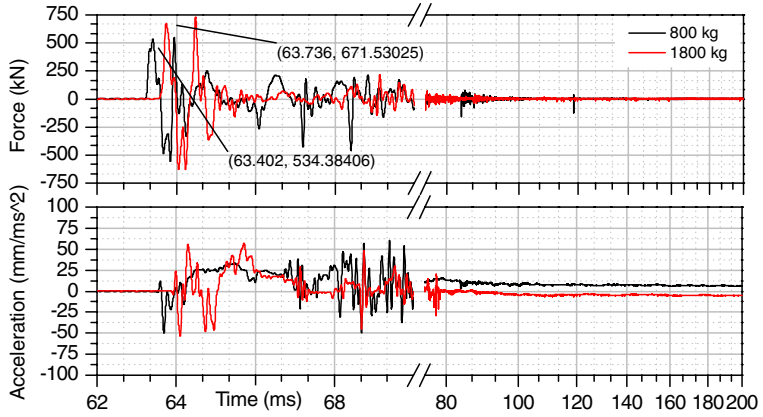

Figure 11. Impactor force and acceleration distributions for different drop masses.
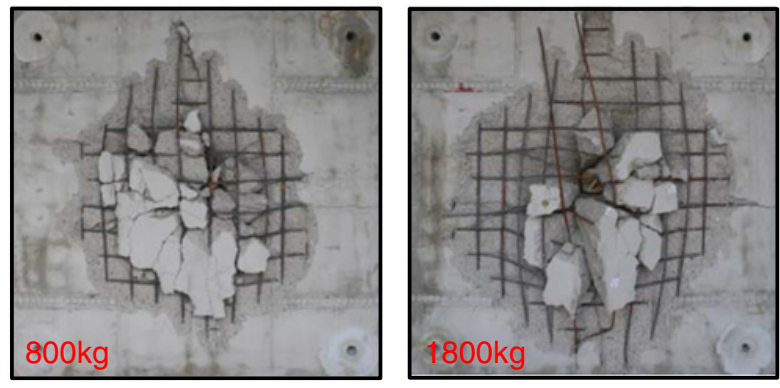

Figure 12. Drop mass influence on the rear side.

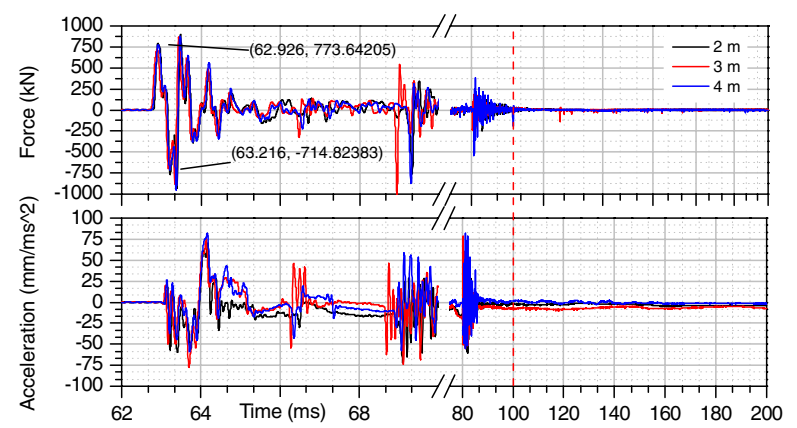

Figure 13. Drop high influence on the damage behavior on the rear side.

$70 \%$ to $50 \%$. This difference should be carried by activated dampers and needs to be considered globally.

Figure 12 again compares the rear sides of the samples for high and low masses. The impactor punched through the whole slab at high mass. This leads to the massive damage with destroyed reinforcement partially reaching its tensile strength. At the left side the impactor is sticking within the slab. The rear side reinforcement is moderately deformed but carries the resultant energy.

\subsection{Drop high variation}

By increasing the drop height the energy and velocity also increases. At this sample configuration three steps at 2, 3 and $4 \mathrm{~m}$ were tested which led to energies of 15 ; 24 and $32 \mathrm{~kJ}$ at impactor speeds of $6.3,7.7$ and $8.6 \mathrm{~m} / \mathrm{s}$. The slab thickness is $300 \mathrm{~mm}$ in all cases. The starting times in Fig. 13 were adjusted to fit the impulse start of the $2 \mathrm{~m}$ data. It is shown that the velocity increase does 


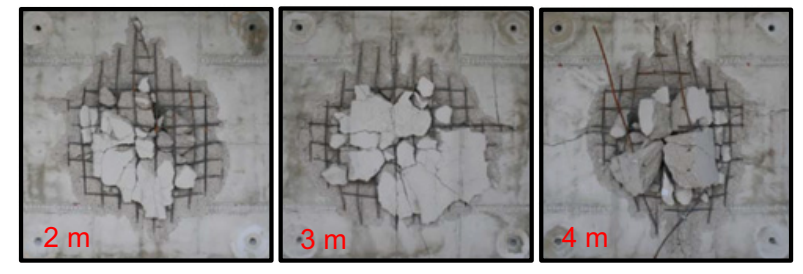

Figure 14. Drop high influence to the damage behavior on the rear side.

not really affect the mass increase or the impulse shape. The amplitudes vary about 5\% around a mean value of $750 \mathrm{kN}$. The impactor tip accelerations vary from 57,49 to $34 \mathrm{~mm} / \mathrm{ms}^{2}$ for heights of 2,3 , and $4 \mathrm{~m}$ which is significant but not fully analyzed yet.

Comparing the rear sides for variable drop heights shows the large indentation at the right side. The high energy at 4 meters again leads to a total punching with activating the rescue dampers. Nevertheless is the data valid during the most relevant first few milliseconds. The energy is close to the energy at the higher drop mass of $1800 \mathrm{~kg}$ and with ignoring the thinner slab the damaged shape rear sides are similar to each other. Comparing with 2 and $3 \mathrm{~m}$ drop height the lower rebar layer is cracked exciting the tensile strength of the steel.

\section{Conclusions}

The remarks given show the relevance of knowing in detail what is going on while measuring data in high speed processes. The theoretical deduction of fundamental times and frequencies are the most important factor establishing a valid measuring concept for such experiments. The knowledge of the sample behavior and its interaction with the test facility leads to much better understanding and interpreting of the sometimes as noise devalued data.
A sample interpretation of the data was done by only two measured values of the impactor since some kind of static spare force are most relevant for civil engineers. In real this restricted view are not valid since much more parameters are affected by the sample-facility interaction. For example the force measurement between impactor and drop masses are affected by cross forces since bending was not considered until now. To compensate this, additional sensors need to be integrated.

The three examples show the difficulties to distinguish between the three main parameters energy, mass and velocity and there influence to the damage behavior. Even to define the term "damage" is not really easy in this type of experiment and needs more consideration. Keeping this in mind, experiments without extensive demolition could give more information about the load capacity of the sample than experiments with energy overloads. To understand this more in detail, extensive numerical simulation of the experiment and the facility are in process leading to improved measure and evaluation concepts for the next series.

\section{References}

[1] P. Bischoff, S. Perry, Materials and Structures 24 (1991)

[2] L. J. Malvar, C. A. Ross, ACI Materials Journal 95 (1998)

[3] U. Häussler-Combe, T. Kühn, International Journal of Impact Engineering (2012)

[4] T. Kühn, U. Häußler-Combe, WCCM proceedings 10 (2012)

[5] B. Beckmann, A. Hummeltenberg, T. Weber, M. Curbach, Structural Engineering International, 22 (2012)

[6] T. Kühn, B. Beckmann, A. Hummeltenberg, M. Curbach, fib proceedings Tel Aviv (2013) 